\title{
A New Wavelet Threshold Function and Denoising Application
}

\author{
Lu Jing-yi, ${ }^{1,2}$ Lin Hong, ${ }^{2}$ Ye Dong, ${ }^{1}$ and Zhang Yan-sheng ${ }^{1,2}$ \\ ${ }^{1}$ School of Electrical Engineering and Automation, Harbin Institute of Technology, Harbin 150001, China \\ ${ }^{2}$ Faculty of Electricity and Information Engineering, Northeast Petroleum University, Daqing 163318, China \\ Correspondence should be addressed to Ye Dong; yedong@hit.edu.cn
}

Received 10 December 2015; Revised 19 March 2016; Accepted 13 April 2016

Academic Editor: Huiyu Zhou

Copyright (c) $2016 \mathrm{Lu}$ Jing-yi et al. This is an open access article distributed under the Creative Commons Attribution License, which permits unrestricted use, distribution, and reproduction in any medium, provided the original work is properly cited.

\begin{abstract}
In order to improve the effects of denoising, this paper introduces the basic principles of wavelet threshold denoising and traditional structures threshold functions. Meanwhile, it proposes wavelet threshold function and fixed threshold formula which are both improved here. First, this paper studies the problems existing in the traditional wavelet threshold functions and introduces the adjustment factors to construct the new threshold function basis on soft threshold function. Then, it studies the fixed threshold and introduces the logarithmic function of layer number of wavelet decomposition to design the new fixed threshold formula. Finally, this paper uses hard threshold, soft threshold, Garrote threshold, and improved threshold function to denoise different signals. And the paper also calculates signal-to-noise (SNR) and mean square errors (MSE) of the hard threshold functions, soft thresholding functions, Garrote threshold functions, and the improved threshold function after denoising. Theoretical analysis and experimental results showed that the proposed approach could improve soft threshold functions with constant deviation and hard threshold with discontinuous function problems. The proposed approach could improve the different decomposition scales that adopt the same threshold value to deal with the noise problems, also effectively filter the noise in the signals, and improve the SNR and reduce the MSE of output signals.
\end{abstract}

\section{Introduction}

At present, the speech signal processing techniques have been used in many areas. Denoising is the key to speech signal processing technology, which is the process of removing noise to the maximum extent of noise to restore the original signals [1]. It has become an indispensable link in speech signal processing. Among the methods of denoising, the traditional one is usually based on Fourier transform. In contrast, the wavelet transform is much more automatically adaptive to the requirement of time-frequency signal analysis [2] because any detail of the signal can be captured, and the wavelet transform can solve the problems that Fourier transform cannot in dealing with the nonstationary signals. Using wavelet transform in signal processing is the process of the partial transformation of the spatial domain and frequency domain. Thus, we can get useful information accurately from it with noise. Now, denoising methods can be commonly divided into the following kinds: the modulus maxima denoising method, the correlation denoising method, and the wavelet threshold denoising method [3].
Modulus maxima denoising method is based on the different characteristics of view when the signal and noise models at multiple scales of space maxima are different. Remove those magnitude scales which increase with decreasing extreme value point and then reconstruct these extreme points in order to achieve the elimination of noise [4]. The modulus maxima method in addition to the noise effect is better than any other method when mixed with white noise and singular information is significant, but the amount of calculation is really huge [2]. Correlation denoising method achieves its aim by containing noise signals after wavelet transform so that the correlation degree of the wavelet coefficients of the original part and the noise part on each scale is different. The calculation procedure of this method is complex [5].

Wavelet threshold denoising method was proposed by American scholar Donohue. The method is simple to calculate and the noise can be suppressed to a large extent. At the same time, singular information of the original signal can be preserved well, so it is a simple and effective method [6]. 
Whether wavelet threshold denoising method is good or bad depends on two decisive factors. One is threshold $\lambda$ and the other important factor is the selection of the threshold function [7]. As the most basic threshold function, both hard and soft threshold functions have their own defects. The breakpoint problem of the hard threshold function makes it have no continuity [8]. In the process of reconstructing speech signal, proneness to volatility and pseudo-Gibbs effect will make the speech distorted while the hard threshold function can keep singular information of the original signal. And the constant deviation problem which is born with the soft threshold function cannot be overcome by itself, which means that the reconstructed signal is too smooth and there are constant deviations compared with the original signals. All the abovementioned defects cause part of the high frequency signal information to be missing, which influence the final processing results [9].

In order to overcome the defects of hard and soft thresholding function perspective, this paper constructs an improved wavelet threshold function by increasing the adjustment factor. In order to overcome the problem that the same threshold is used to deal with the different decomposition scales, this paper designs a new fixed threshold formula by introducing the logarithmic functions of layer number of wavelet decomposition.

\section{The Principle of Wavelet Threshold Denoising}

In practical cases, noise signals usually appear as high frequency signals in signal processing, but useful signals appear as either low frequency or more smooth signals. The signals with noise have the features of the above, so when the signals are decomposed by wavelet, the signals with noise in the high frequency wavelet coefficients, through threshold quantization threshold processing high frequency wavelet coefficients to reconstruct the signals, can eliminate the noise with the signals. One dimensional signal denoising process is as follows: the one dimensional signal is decomposed by wavelet decomposition, selecting threshold and threshold function to quantify the high frequency coefficients of wavelet decomposition and reconstruct the one dimensional wavelet. The key factors affecting the quality of denoising are denoising threshold and the selection of threshold function [10].

\section{Wavelet Threshold Function}

3.1. Classical Threshold Function. The hard threshold function sets the decomposition coefficient to zero which is less than the threshold value under different scale spaces and reserves the decomposition coefficient which is greater than the threshold at the same time [11]. This method does not change the local properties of the signal, but because of the discontinuity, it leads to a certain fluctuation in the reconstruction of the original signal. Consider

$$
\overline{\omega_{j, k}}= \begin{cases}\omega_{j, k}, & \left|\omega_{j, k}\right| \geq \lambda, \\ 0, & \left|\omega_{j, k}\right|<\lambda .\end{cases}
$$

The soft threshold function is to select the specified threshold value of the decomposition coefficient to zero. After the algorithm, the decomposition coefficient is coherent, but it loses a part of the high frequency coefficients above the threshold [12]. Consider

$$
\overline{\omega_{j, k}}= \begin{cases}\operatorname{sgn}\left(\omega_{j, k}\right)\left(\left|\omega_{j, k}\right|-\lambda\right), & \left|\omega_{j, k}\right| \geq \lambda \\ 0, & \left|\omega_{j, k}\right|<\lambda\end{cases}
$$

Parameter $\overline{\omega_{j, k}}$ is the representation of estimated wavelet coefficients, parameter $\omega_{j, k}$ is the representation of the wavelet coefficients after decomposition, $\lambda$ is the representation of threshold, and $\operatorname{sgn}(*)$ is the symbolic piecewise function in the above two formulas [13].

In order to overcome the shortcomings of above two methods, GaoHong Ye proposed Garrote threshold function in which the denoising effect is the best compared with above two kinds of traditional threshold functions and it is better in continuity with the expressions such as

$$
\overline{\omega_{j, k}}= \begin{cases}\omega_{j, k}-\frac{\lambda^{2}}{\omega_{j, k}}, & \left|\omega_{j, k}\right| \geq \lambda, \\ 0, & \left|\omega_{j, k}\right|<\lambda .\end{cases}
$$

3.2. The Improved Threshold Function. The continuity in the soft threshold function is much better, but it has a constant deviation. So, in order to overcome its shortcomings, the soft and hard threshold algorithms are compromised process by the literature; the semisoft threshold function [14] (4) has been shown in the formula

$$
\overline{\omega_{j, k}}= \begin{cases}\operatorname{sgn}\left(\omega_{j, k}\right)\left(\left|\omega_{j, k}\right|-T \lambda\right), & \left|\omega_{j, k}\right| \geq \lambda, \\ 0, & \left|\omega_{j, k}\right|<\lambda .\end{cases}
$$

Among the soft and hard threshold functions, the value of $T$ is taken between 0 and 1 . Even if the result of semisoft threshold function is between them, the value of $T$ is fixed. So, there will still be fixed bias. Consider

$$
\begin{aligned}
& \overline{\omega_{j, k}} \\
& = \begin{cases}\operatorname{sgn}\left(\omega_{j, k}\right)\left\{\left|\omega_{j, k}\right|-\frac{\lambda}{\exp ^{3}\left[\alpha\left(\left|\omega_{j, k}\right|-\lambda\right) / \lambda\right]}\right\}, & \left|\omega_{j, k}\right| \geq \lambda, \\
0, & \left|\omega_{j, k}\right|<\lambda .\end{cases}
\end{aligned}
$$

The threshold function is proposed in this paper which is expressed in formula (5). The adjustment factor of the new function is different from the semisoft threshold function. It consists of a complex exponential function $\exp ^{-3}\left[\alpha\left(\left|\omega_{j, k}\right|-\right.\right.$ $\lambda) / \lambda$ ] which has more adaptability; $\alpha$ is the normal number which can be adjusted freely and the values of $\alpha$ are different with the different signal. When $\left|\omega_{j, k}\right|=\lambda, \overline{\omega_{j, k}}=0$ and when $\left|\omega_{j, k}\right| \rightarrow \lambda, \overline{\omega_{j, k}} \rightarrow 0$. Therefore, continuously in place of $\lambda$, the improved threshold function has the characteristics of soft threshold function; when $\left|\omega_{j, k}\right| \rightarrow \infty, \overline{\omega_{j, k}} \rightarrow \omega_{j, k}$ improved threshold function based on $\overline{\omega_{j, k}}=\omega_{j, k}$ as the asymptotic line; it can be seen that, with the increase of $\omega_{j, k}$, $\omega_{j, k}$ will gradually be close to $\omega_{j, k}$; when $\omega_{j, k}$ becomes infinite, 


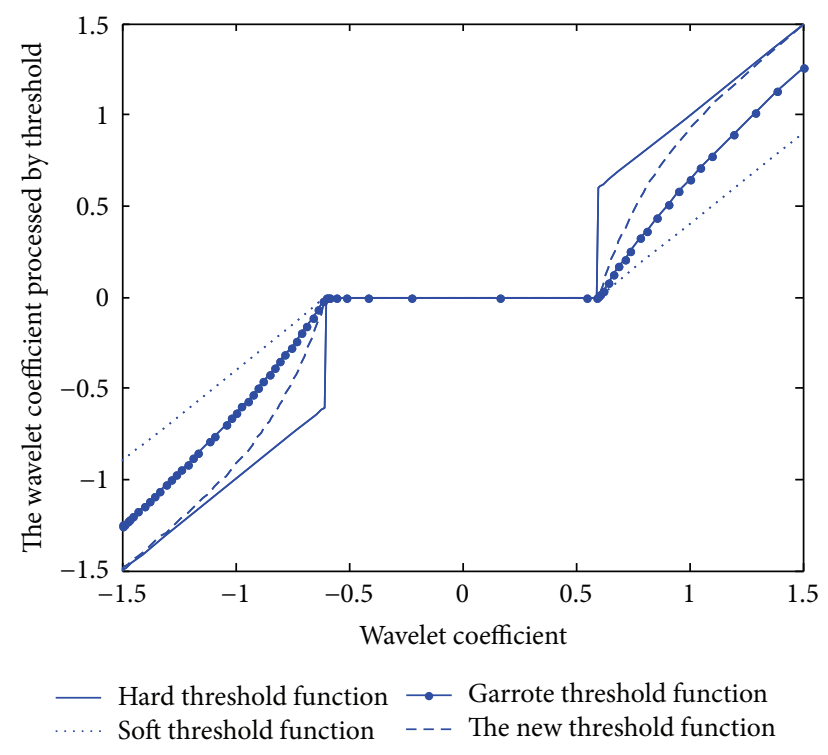

FIGURE 1: Comparison of different thresholding functions.

$\omega_{j, k}$ and $\omega_{j, k}$ can be approximated as equal. That will not only reduce the discreteness of the hard threshold function, but can also avoid the constant problem in soft threshold function at the same time. The variables $\alpha$ in the formula are also really important, and the change of the value of $\alpha$ can affect the noise directly. When $\alpha=0$, the function will express soft threshold function and when $\alpha=\infty$, the function will express hard threshold function.

Take some simple numerical parameters, and then show the soft, hard, Garrote, and new threshold function in the form of graphics intuitive. Here, the parameter threshold function of each take $\lambda=0.6, \alpha=1$, and $-1.5 \leq \omega_{j, k} \leq 1.5$.

As shown in Figure 1, the hard threshold function is not continuous at the threshold point, so that there are fluctuations in the recovery of the original signal. Compared with the hard threshold function, although there are no discontinuity problems in the soft threshold function, there are still some differences between the original signal and the reconstructed signal, because of the constant problems inherent in the soft threshold function.

The new threshold function has continuity and when the coefficient is reaching $\lambda$ it gradually becomes close to zero. Put the hard threshold function curve as the asymptote, and it is reaching the hard threshold results more than Garrote threshold function. Therefore, the new threshold function overcomes the deficiencies of discontinuous and constant deviation, taking the advantages of the three functions. So, in theory, denoising effect is better.

\section{The Improved Fixed Threshold $\lambda$}

The threshold value $\lambda$ is a very important parameter in the wavelet threshold denoising algorithm, and the performance of wavelet denoising will directly be affected by threshold selection. There are some thresholds, such as rigrsure, heursure, sqtwolog, and minimaxi [15]. The fixed threshold is
TABLE 1: Result of SNR and MSE after Heavy sine's denoising.

\begin{tabular}{lcccc}
\hline & $\begin{array}{c}\text { Hard } \\
\text { threshold }\end{array}$ & $\begin{array}{c}\text { Soft } \\
\text { threshold }\end{array}$ & $\begin{array}{c}\text { Garrote } \\
\text { threshold }\end{array}$ & New threshold \\
\hline SNR & 29.5306 & 27.4918 & 30.7351 & 31.2172 \\
MSE & 0.2032 & 0.3249 & 0.1539 & 0.1378 \\
\hline
\end{tabular}

used commonly. Its expression is $\lambda=\sigma_{n} \sqrt{2 \ln N}, \sigma_{n}$ is the noise standard variance, and $N$ is the length of the signal. The same threshold value $\lambda$ is used to deal with the different decomposition scales $j$, so the enhancement effect is not ideal. This paper proposes the improved fixed threshold based on the fixed threshold. Its expression is $\lambda=\sigma_{n} \sqrt{2 \ln N} / \log _{2}(j+$ 1 ); with the increasing of decomposition scale $j$, the value of the improved threshold $\lambda$ is gradually reduced. And it is consistent with the propagation characteristics of the noise in different scales of the wavelet transform to ensure that the denoising is effective. The improved threshold method can make sure that the information of useful signal has a great extent.

\section{The Experiment and Comparison}

There are two important bases for judging the effect of denoising. One indicator is SNR, and another is MSE [12]. SNR is the proportion of the energy of the signal and noise in the noise signal. It is proportional to the relationship with denoising effect. MSE and denoising effect have an inversely proportional relationship. It is a means to measure average errors [13].

5.1. Simulation Experiment. We can do the experiments using Heavy sine and Bumps curve in Matlab environment and use the soft, hard, Garrote, and the improvement of the threshold function method to deal with two kinds of curves. The number of layers is the five. Meanwhile, use Daubechies wavelet as wavelet basis. The processing results are treated by using the SNR and MSE.

Four kinds of methods are used for the sine Heavy curves to do the noise removal test. The results are shown in Figure 2.

Through the results of their own processing, they show that the distortion of the sine Heavy curve is in the process of soft and hard threshold function. While the results of Garrote threshold function and the new methods of processing are more ideal, whose effects are better cannot be identified with the naked eyes, which need to analyze SNR and MSE.

By Table 1, the sine Heavy curve can be processed by the proposed method in this paper, and the SNR is 31.2172. Not only is the SNR of the proposed method 0.0571 times higher than that of the hard threshold function, but also is 0.1355 times higher than that of soft threshold function. At the same time, the Garrote threshold function increases 0.0156 times. The minimum mean square error is 0.3218 , which is 0.5758 times less than the hard threshold function, 0.1378 times less than the soft threshold function, and 0.1046 times less than the Garrote threshold function. So the proposed method is best at reducing the noise effect. 

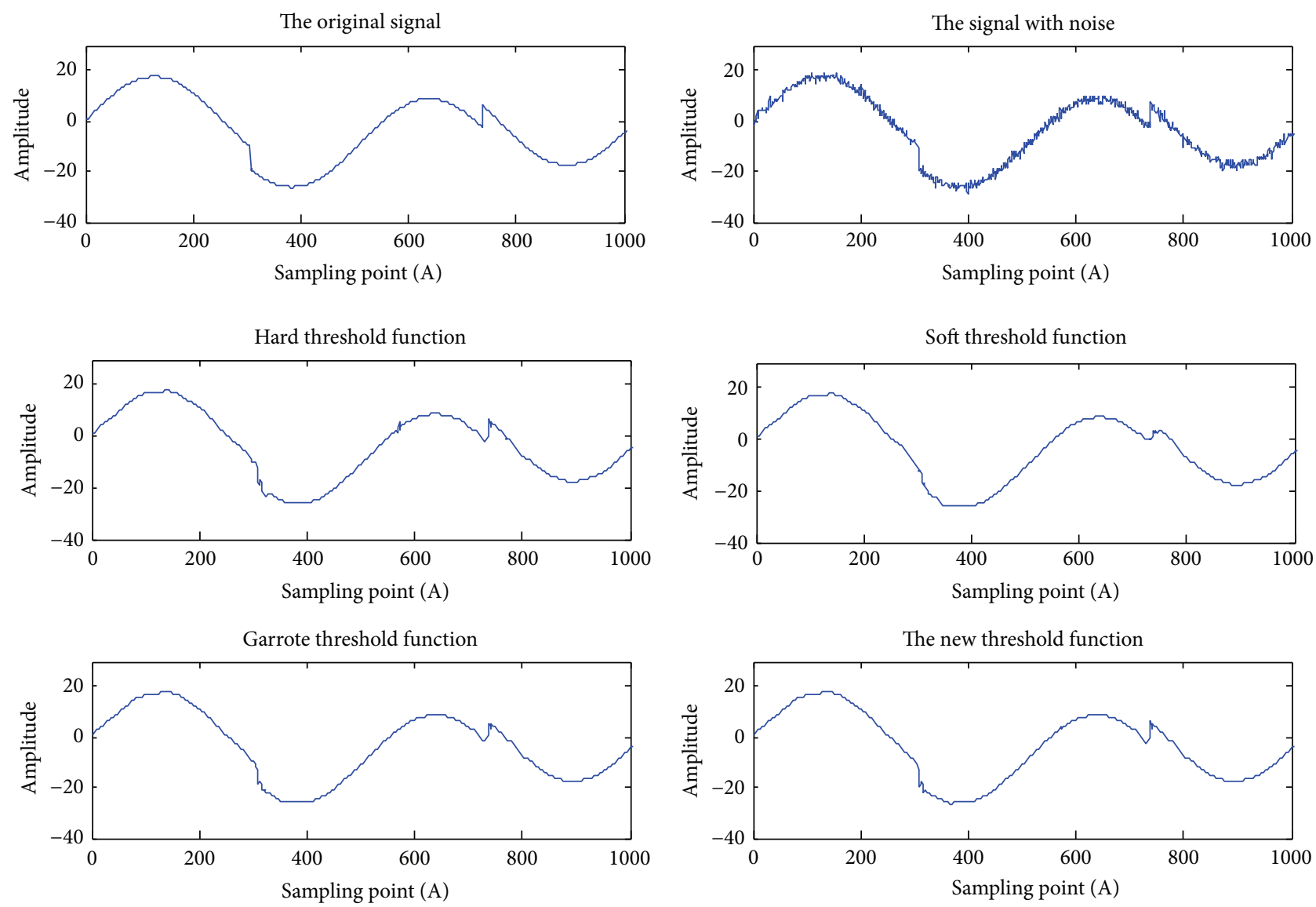

FIGURE 2: Result of Heavy sine processing.

TABLE 2: Result of SNR and MSE after Bumps denoising.

\begin{tabular}{lcccc}
\hline & $\begin{array}{c}\text { Hard } \\
\text { threshold }\end{array}$ & $\begin{array}{c}\text { Soft } \\
\text { threshold }\end{array}$ & $\begin{array}{c}\text { Garrote } \\
\text { threshold }\end{array}$ & New threshold \\
\hline SNR & 21.4198 & 16.0230 & 26.0354 & 27.0029 \\
MSE & 1.6647 & 5.7680 & 0.5752 & 0.4603 \\
\hline
\end{tabular}

We look at the results of the Bumps curves with noise by using four methods, which can be seen from Figure 3; the denoising effects of the hard threshold method are still not satisfactory. After processing, the signal has lost some of the information and it is still unable to see the Garrote threshold function and the new function proposed in this paper. Table 2 shows that the method is more effective.

By Table 2, the SNR of the proposed method is as high as 27.0029 , compared with the hard threshold method, which is 0.2606 times higher and 0.6852 times higher than that of soft threshold method. At the same time, it is 0.0371 times higher than the Garrote threshold method. The minimum mean square error is 0.4603 , which is 0.7234 times less than the hard threshold function, 0.9201 times less than the soft threshold function, and 0.1997 times less than the Garrote threshold function. As a result, the proposed method in this paper is still the best of the four methods of the Bumps process.

5.2. The Actual Speech Signal Denoising Experiments. Through the comparison and analysis of sine Bumps and Heavy curves, the advantages of the proposed method in this paper are proved. The noise of speech signals collected in the field is more convincing. The original signal is a relatively pure female speech signal, in order to facilitate the observation of the effect of noise, which intercepts the experiments that were conducted to join the noise of original signal, with four kinds of methods of the noisy speech processing, wavelet decomposition layer choices of five layers, and selection of Daubechies wavelet as mother wavelet.

As shown in Figure 4, different threshold functions are obtained by different processing results. Among them, the soft threshold function is the most unsatisfactory, which is a distortion phenomenon. The hard threshold function can retain most of the original signal singular information retained, but the signal is significantly shaken in the process of reconstruction. Garrote threshold function and the improved function of the denoising effect are the greatest degree of 

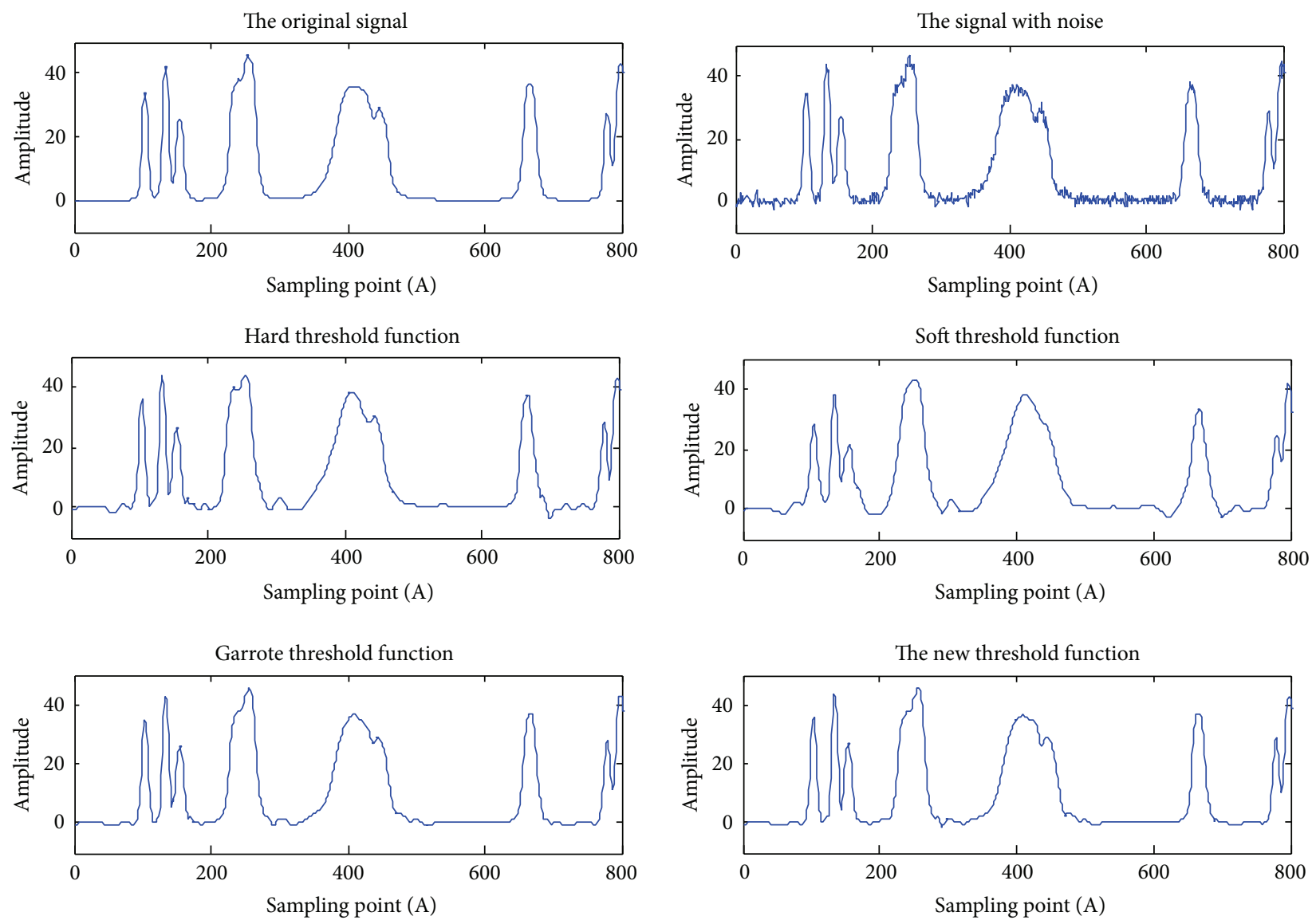

Figure 3: Results of Bumps processing.

TABLE 3: Result of SNR and MSE after speech signal's denoising.

\begin{tabular}{lcccc}
\hline & $\begin{array}{c}\text { Hard } \\
\text { threshold }\end{array}$ & $\begin{array}{c}\text { Soft } \\
\text { threshold }\end{array}$ & $\begin{array}{c}\text { Garrote } \\
\text { threshold }\end{array}$ & New threshold \\
\hline SNR & 10.8765 & 9.5528 & 10.9919 & 11.4036 \\
MSE $\left(10^{-4}\right)$ & 4.5911 & 6.2272 & 4.4707 & 4.0664 \\
\hline
\end{tabular}

reduction of the original signal characteristics. In order to determine which method of noise performance is best, we need to use the SNR and the MSE of these two measures to determine it.

By Table 3, the SNR value of the proposed method in this paper was 11.4036, which was 0.0484 times higher than that of the hard threshold, 0.1937 times higher than that of the soft threshold, and 0.0374 times higher than the Garrote threshold function. The MSE of the proposed method in this paper is 4.0664. The MSE of the modified threshold function is 0.1142 times lower than the hard threshold function, 0.3469 times lower than the soft threshold function, and 0.0904 times less than the Garrote threshold function. Through SNR and MSE, the two measures are further explained in which the performance of the new threshold function is better than the other 3 kinds of threshold functions.
5.3. The Shot Signal Denoising Experiment. In order to further verify the superiority of the improved threshold function denoising effect, this paper constructed the passive acoustic data acquisition system by using PXIe1082 NI, PXIe8820 NI, PXIe4492 NI, and AWA14604 sensors. In the room (15 m, 7 m, and $4 \mathrm{~m}$ ), we used a computer to control a high fidelity sound box as the simulation gun. The experiment was carried out in advance to measure the position. In order to observe the effect of denoising, we intercepted a section of firing signal to carry out the experiment. The simulation results are shown in Figure 5.

As shown in Figure 5, soft threshold function is not the most ideal. Because there is a distortion of the phenomenon, and it is hard to see the differences among hard threshold function, Garrote threshold function, and the proposed method in this paper. To determine which method is the optimal denoising performance with the SNR and MSE, the simulation results are shown in Table 4.

In Table 4 , the SNR of the output signal by the proposed method in this paper is 8.1647, which is the largest, and it is 1.0901 times higher than that of the hard threshold, 2.1388 times higher than that of the soft threshold, and 1.3127 times higher than that of the Garrote threshold function. The MSE of the proposed method in this paper is 0.0028 , and the MSE 

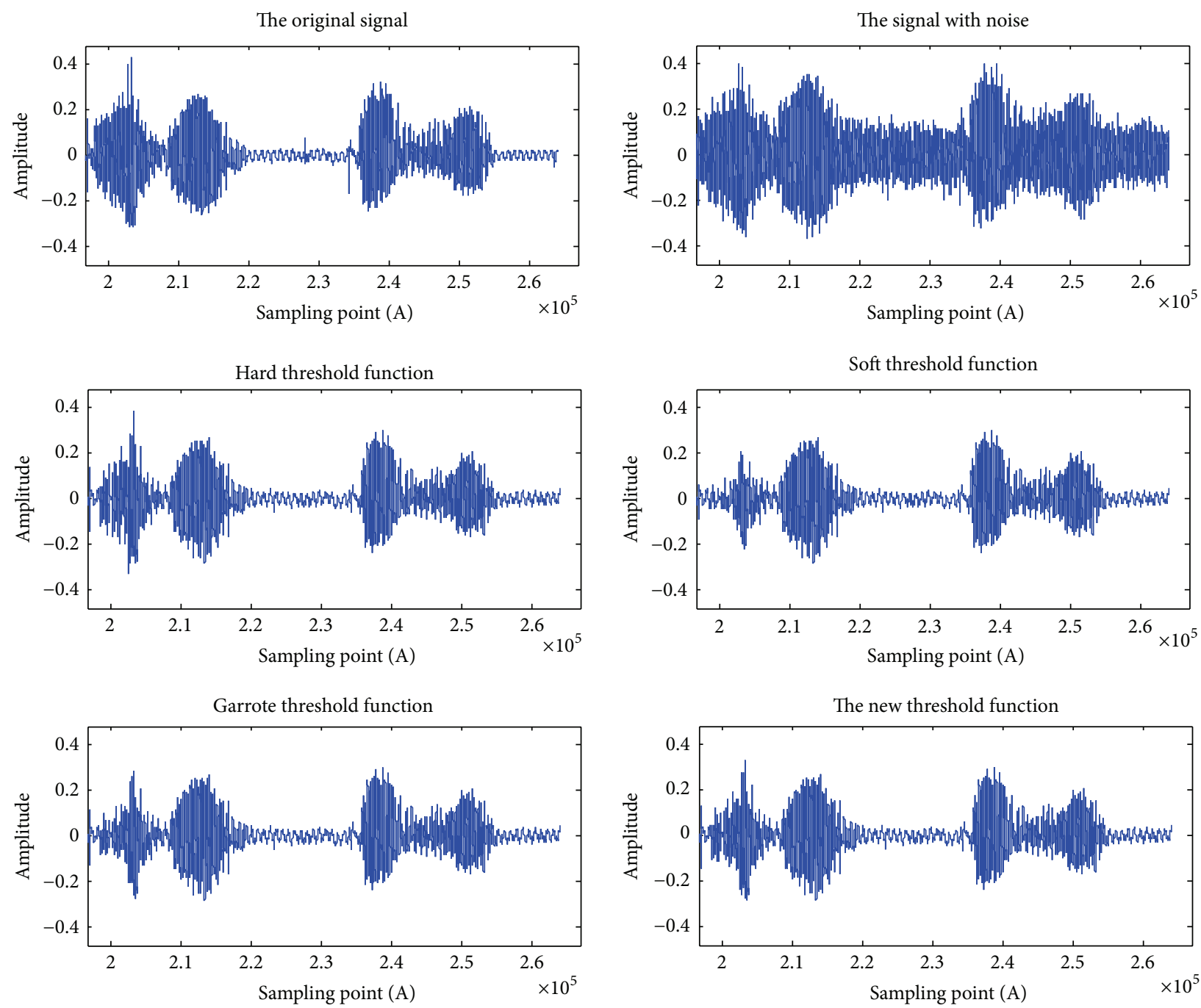

FIGURE 4: Results of noise speech signal processing.

TABLE 4: SNR and MSE of the shot signal denoising.

\begin{tabular}{lcccc}
\hline & $\begin{array}{c}\text { Hard } \\
\text { threshold }\end{array}$ & $\begin{array}{c}\text { Soft } \\
\text { threshold }\end{array}$ & $\begin{array}{c}\text { Garrote } \\
\text { threshold }\end{array}$ & New threshold \\
\hline SNR & 7.4924 & 3.8277 & 6.2220 & 8.1674 \\
MSE & 0.0033 & 0.0076 & 0.0044 & 0.0028 \\
\hline
\end{tabular}

of the improved method is 0.8485 times lower than that of the hard threshold function, which is 2.7143 times lower than that of the soft threshold function and 0.6364 times less than that of the Garrote threshold function.

By means of SNR and MSE, these two indicators, we can verify the stability of the denoising performance of the proposed method in this paper.

\section{Conclusion}

This paper first introduced the wavelet threshold denoising in the traditional threshold function and then proposed the new wavelet threshold function according to the function curve of the denoising performance analysis to improve the function, through the increase in soft threshold function adjustment factor. This paper studied the fixed threshold and introduced the logarithmic function of layer number of wavelet decomposition to design a new threshold formula. The proposed method can make the threshold estimation more accurate and lead to better denoising effects. Theoretical analysis and experimental results showed that using the proposed method in this paper can improve the SNR of the output signal and reduce the MSE of the output signal, improve the soft threshold function with constant deviation and the hard threshold with discontinuous function problem, improve the different decomposition scales to adopt the same threshold value to deal with the noise problem, and verify that the proposed method is practical, effective, and feasible, compared with the traditional method of denoising threshold function that the proposed approach can effectively filter the noise in the signal. 

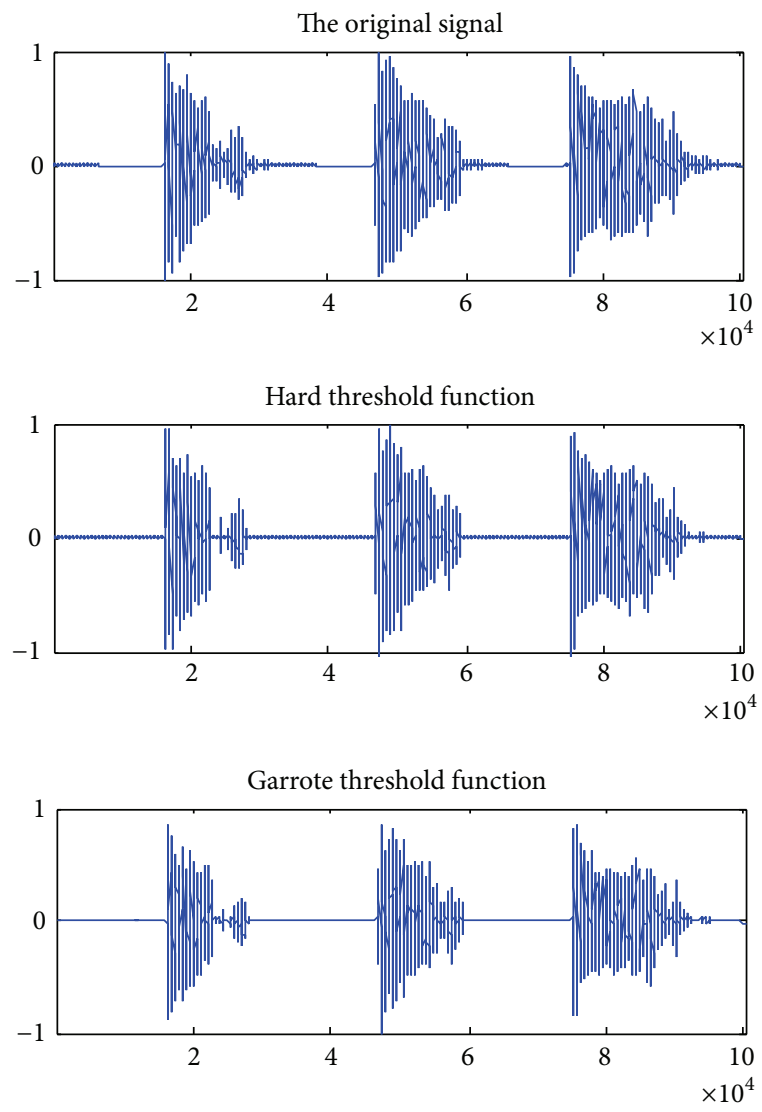

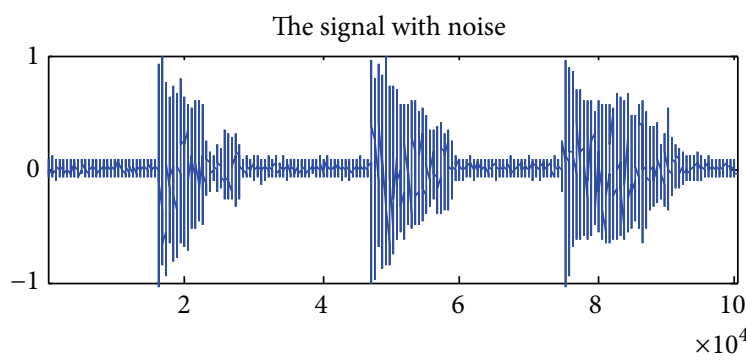

Soft threshold function
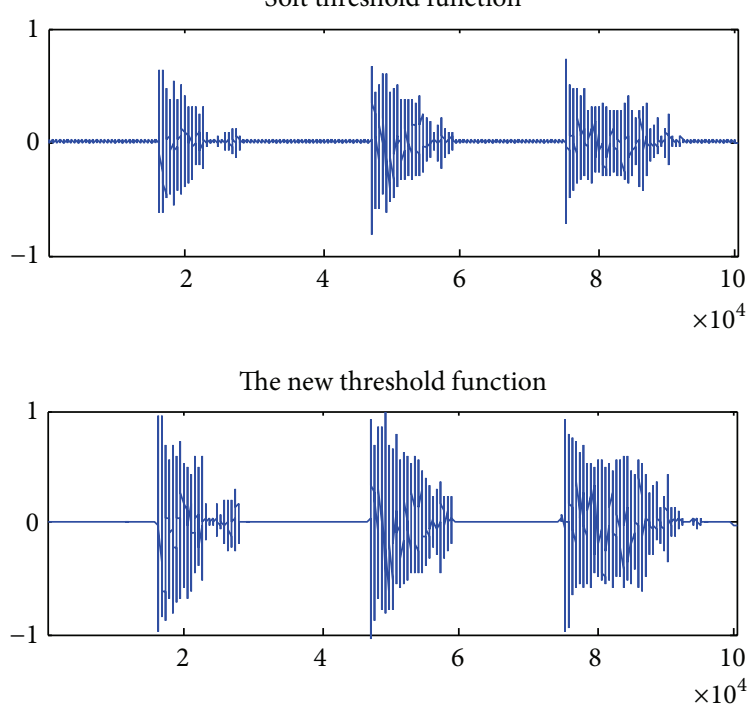

FIgURE 5: Processing results of noisy shot signal.

\section{Competing Interests}

The authors declare that they have no competing interests.

\section{Authors' Contributions}

Lu Jingyi participated in the data collection, analysis, and algorithm simulation. Lin hong and Ye Dong participated in the concept, design, and interpretation and commented on the paper. A substantial amount of Zhang Yansheng's contribution was to the draft writing and critical revision of this paper. All authors read and approved the paper.

\section{Acknowledgments}

This work is partially supported by the National Nature Science Foundation of China (Grant no. 51075095) and the Nature Science Foundation of Heilongjiang Province (Grant no. E201045).

\section{References}

[1] W. B. Xu, X. C. Wu, and J. P. Xing, "A new method for wavelet threshold denoising," Journal of LanZhou JiaoTong University, vol. 31, no. 3, pp. 120-124, 2012 (Chinese).

[2] B. L. Jin, H. Li, N. J. Zhao et al., "A new denoising algorithm for wavelet thresholding," Journal of Missile and Missile, vol. 31, no. 1, pp. 167-169, 2011 (Chinese).
[3] T. F. Sanam and C. Shahnaz, "A semisoft thresholding method based on Teager energy operation on wavelet packet coefficients for enhancing noisy speech," EURASIP Journal on Audio,Speech, and Music Processing, vol. 2013, article 25, 2013.

[4] D. L. Donoho, "De-noising by soft-thresholding," IEEE Transactions on Information Theory, vol. 41, no. 3, pp. 613-627, 1995.

[5] D. L. Donoho and I. M. Johnstone, "Adapting to unknown smoothness via wavelet shrinkage," Journal of the American Statistical Association, vol. 90, no. 432, pp. 1200-1224, 1995.

[6] D. L. Donoho and I. M. Johnstone, "Ideal spatial adaptation by wavelet shrinkage," Biometrika, vol. 81, no. 3, pp. 425-455, 1994.

[7] C. He, J. Xing, J. Li, Q. Yang, and R. Wang, "A new wavelet thresholding function based on hyperbolic tangent function," Mathematical Problems in Engineering, vol. 2015, Article ID 528656, 9 pages, 2015.

[8] H.-R. Jia, X.-Y. Zhang, and J. Bai, "A continuous differentiable wavelet threshold function for speech enhancement," Journal of Central South University, vol. 20, no. 8, pp. 2219-2225, 2013.

[9] L. Su, G. Zhao, and R. Zhang, "Translation-invariant wavelet de-noising method with improved thresholding," in Proceedings of the IEEE International Symposium on Communications and Information Technology (ISCIT '05), vol. 1, pp. 619-622, October 2005.

[10] A. Phinyomark, C. Limsakul, and P. Phukpattaranont, “Optimal wavelet functions in wavelet denoising for multifunction myoelectric control," ECTI Transactions on Electrical Engineering, Electronics, and Communications, vol. 8, no. 1, pp. 43-52, 2010.

[11] C. He, J. C. Xing, and Q. L. Yang, "Optimal wavelet basis selection for wavelet denoising of structural vibration signal," 
Applied Mechanics and Materials, vol. 578-579, pp. 1059-1063, 2014.

[12] J.-Y. Tang, W.-T. Chen, S.-Y. Chen, and W. Zhou, "Waveletbased vibration signal denoising with a new adaptive thresholding function," Journal of Vibration and Shock, vol. 28, no. 7, pp. 118-121, 2009 (Chinese).

[13] S. Badiezadegan and R. C. Rose, "A wavelet-based thresholding approach to reconstructing unreliable spectrogram components," Speech Communication, vol. 67, pp. 129-142, 2015.

[14] X. Chen, S. Li, and W. Wang, "New de-noising method for speech signal based on wavelet entropy and adaptive threshold," Journal of Information and Computational Science, vol. 12, no. 3, pp. 1257-1265, 2015.

[15] K. L. Yuan, "Wavelet denoising based on threshold optimization method," Engineering Journal of Wuhan University, vol. 48, no. 1, pp. 74-80, 2015. 


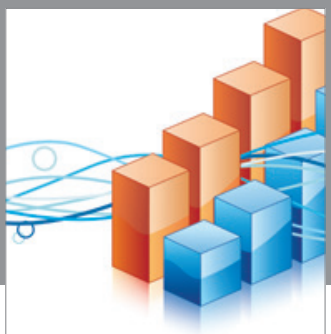

Advances in

Operations Research

vatem alat4

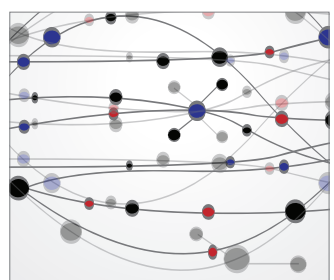

\section{The Scientific} World Journal
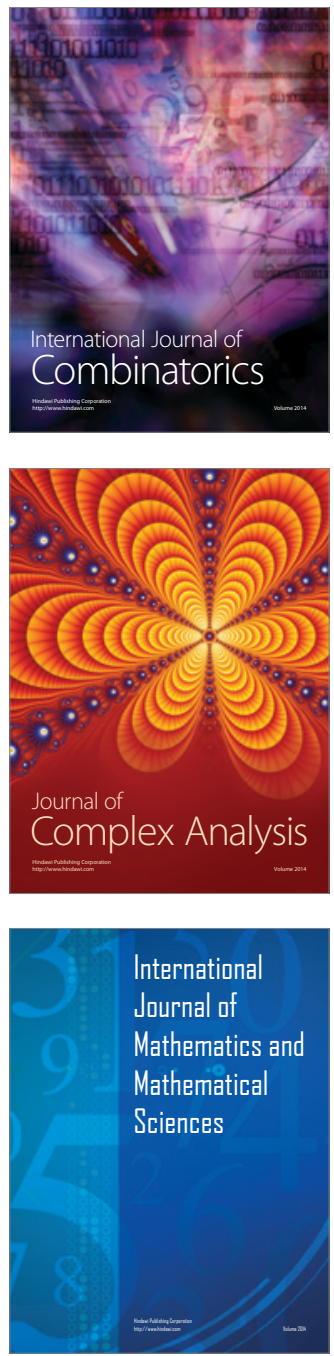
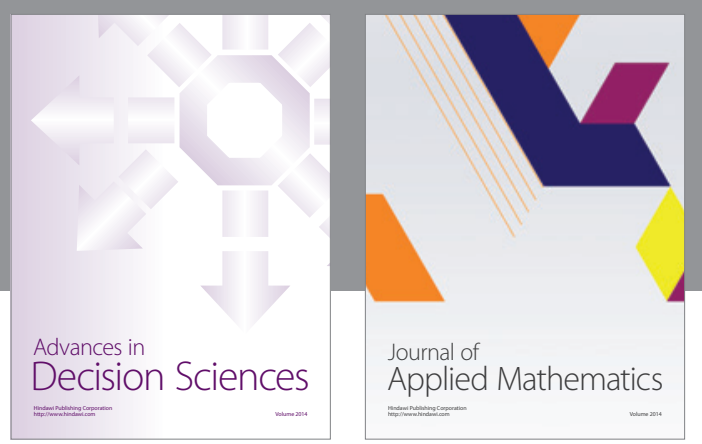

Algebra

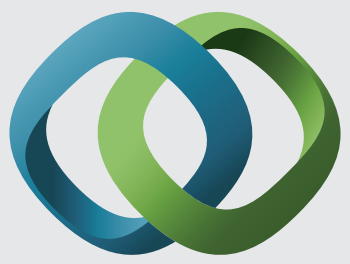

\section{Hindawi}

Submit your manuscripts at

http://www.hindawi.com
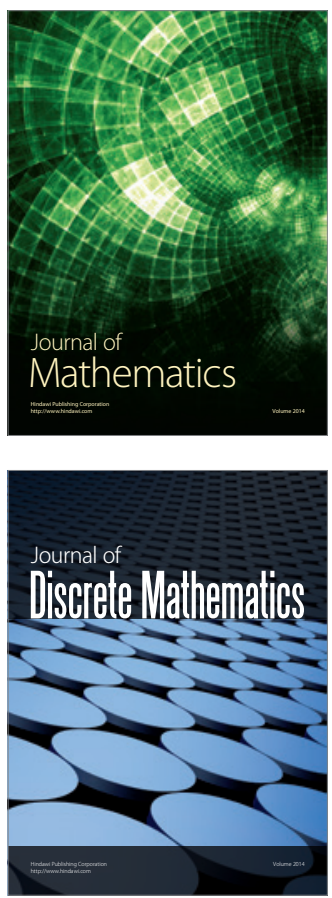

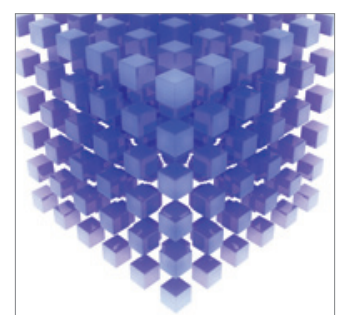

Mathematical Problems in Engineering
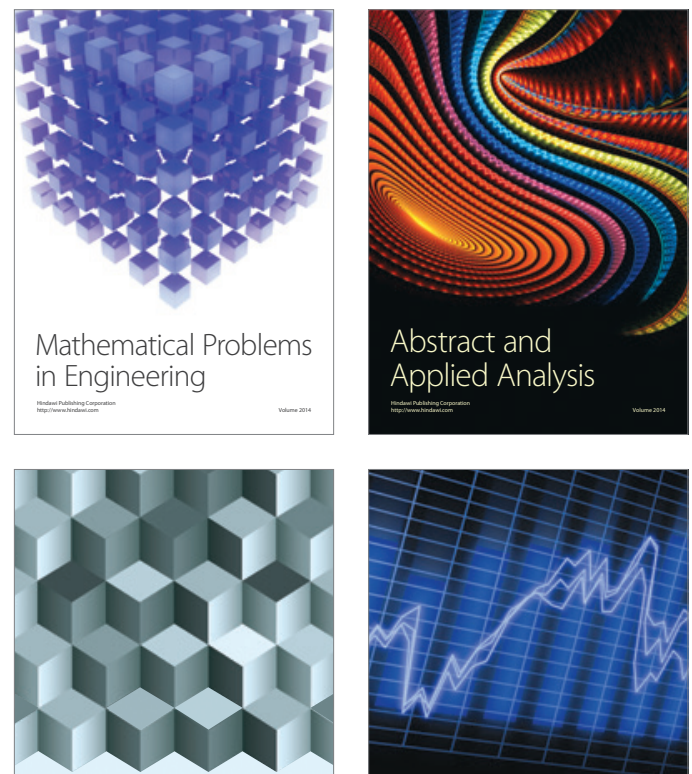

Journal of

Function Spaces

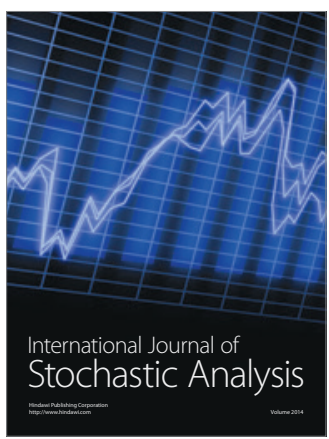

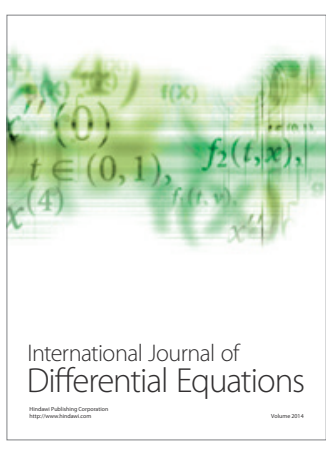
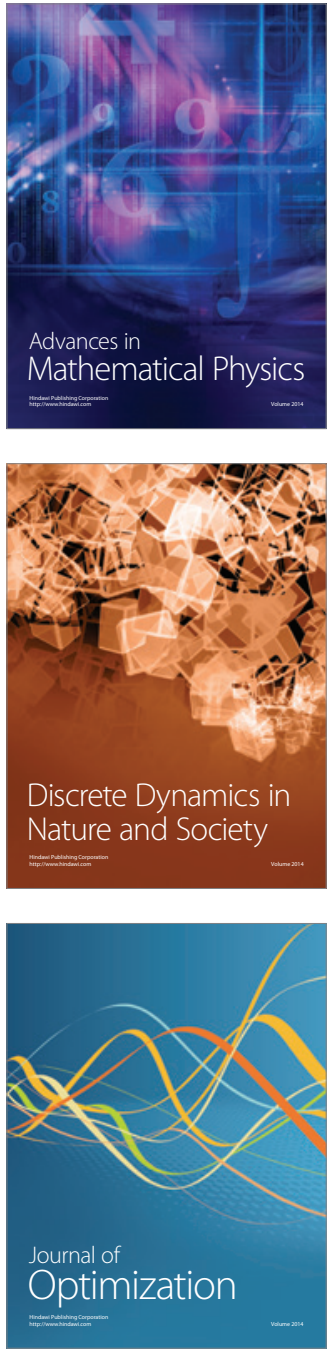